\title{
An investigation of maternal attitudes to breast-feeding in public and breast-feeding duration in four European centres
}

\author{
J. A. Scott ${ }^{1}$, Y. Y. Kwok ${ }^{1}$, K. Synnott ${ }^{2}$, C. A. Edwards ${ }^{3}$ and Members of the INFABIO Research Team \\ ${ }^{1}$ Nutrition and Dietetics, School of Medicine, Flinders University, Adelaide, Australia, ${ }^{2}$ Danone Research - Centre for \\ Specialised Nutrition, Wageningen, The Netherlands and ${ }^{3}$ Human Nutrition Section, Division of Developmental Medicine, \\ University of Glasgow, $U K$
}

There is wide variation in the duration of breast-feeding across Europe, which may be attributed in part to cultural differences in perceptions of the social acceptability of breast-feeding in public. This study aimed to investigate the relationship of maternal attitudes towards breast-feeding in public and breast-feeding duration in four European centres (Glasgow, Scotland; Grenada, Spain; Stockholm, Sweden and Reggio-Emilia, Italy) participating in the EU-funded INFABIO project.

Participants ( $n$ 389) were breast-feeding mothers of healthy, singleton births recruited from maternity wards within $3 \mathrm{~d}$ post partum participating in a longitudinal study investigating the association of diet and lifestyle on gastrointestinal infections and allergy in early infancy. Socio-demographic data and information on infant feeding practices and attitudes were collected by self-completed questionnaires at baseline and selected time points until 12 months of age. Infant feeding attitudes were assessed using the 17-item Iowa Infant Feeding Attitude Scale (IIFAS). Cox's proportional-hazards model was used to identify the risk factors independently associated with a shorter breast-feeding duration.

No significant difference was found in the overall breast-feeding attitudes of mothers between centres, but there were significant differences in a number of individual attitude items. Women in the two Northern European centres were more likely to disagree (Glasgow $90.4 \%$ and Stockholm $91.5 \%$ ) that women should not breast-feed in public places such as restaurants, compared to women in the Southern European centres (Granada 58.3\% and Reggio-Emilia 43.7\%). Mothers who had a negative attitude to breast-feeding in public were less likely to have ever breast fed in public $(\mathrm{OR}=0.06,95 \% \mathrm{CI} 0.02,0.18)$; and those who had never breast fed in public were in turn more likely to discontinue breast-feeding earlier ( $\mathrm{HR}=1.51,95 \% \mathrm{CI} 1.11,2.05)$.

Cultural differences and similarities in maternal breast-feeding attitudes were evident in the four centres. Maternal attitude to breastfeeding in public was a strong predictor of breast-feeding duration independent of maternal socio-demographic characteristics.

The INFABIO project was financially supported by the Commission of the European Communities, specific RTD programme 'Quality of Life and Management of Living Resources', Key Action 1, 'Food, Nutrition and Health'. It does not necessarily reflect its views and in no way anticipates the Commission's future policy in this area. 